\title{
Camera Settings for Dimensional Inspection Using Displacement and Quantization Errors
}

\author{
Kevin L. Crosby ${ }^{\dagger} \star \star$, Christopher C. Yang $\dagger$, Frank W. Ciarallo $\ddagger$, and Michael M. Marefat $\dagger$ \\ $\dagger$ Department of Electrical and Computer Engineering, University of Arizona, Tucson, AZ 85721 \\ $\ddagger$ Department of Systems and Industrial Engineering, University of Arizona, Tucson, AZ 85721 \\ * as of Feb. '97, with Lockheed Martin Engineering and Sciences, 2400 NASA Road One, Houston, TX 77258
}

\begin{abstract}
An important aspect of inspection planning involves determining camera poses based on some criterion. We seek to find camera poses where the effects of displacement and quantization errors are minimal. The mean squared error is formulated, including all dependencies, and minimized to determine an optimal camera pose that satisfies the sensor constraints of resolution, focus, field-of-view, and visibility. Dimensional tolerances for line entities are also formulated and exploited to determine the acceptability of a given camera pose for all entities observed.
\end{abstract}

\section{Introduction}

Upon using a camera as a sensor, it is important to know how to position and orient the camera (often called the sensor setting or the camera pose) to most effectively and efficiently inspect desired object entities, such as circular profiles of holes or dimensions of edges. The criterion for effectiveness include having the entity resolvable, in focus, within the field of view, and unoccluded. Efficiency, on the other hand, deals with how quickly information can be obtained, such as finding the minimum number of sensor settings required to visually inspect each important entity. Often a tradeoff exists between effectiveness and efficiency.

Effectiveness and efficiency are achieved through the $\mathrm{Vi}$ sual Inspection Planning with Error Reduction (VIPER) system, which is summarized by the flowchart shown in Figure 1. The scope of this paper is to determine optimal camera poses and to satisfy tolerance criteria.

Several researchers have previously worked on the vision sensor planning topic. The VIO (Vision Illumination Object) system by Niepold, et al [4], the HEAVEN system by Sakane, et al [5], and the ICE (Illumination Control Expert) system by $\mathrm{Yi}$, et al [10] determine a camera position on a geodesic dome or tessellated sphere surrounding the object, and orient the camera toward an object reference point. Cowan and Kovesi [2] formulate the resolution, focus, and field-of-view constraints independent of orientation. The MVP (Machine Vision Planner) system by Tarabanis, et al, formulates the resolution, focus, and fieldof-view constraints with respect to the orientation [6], develops algorithms for deternining unoccluded regions (visibility constraints) [7], and chooses a camera pose in the Euclidean center of the constraints.

Yang, et al [9] modeled the errors involved with camera displacement and quantization for line entities to determine

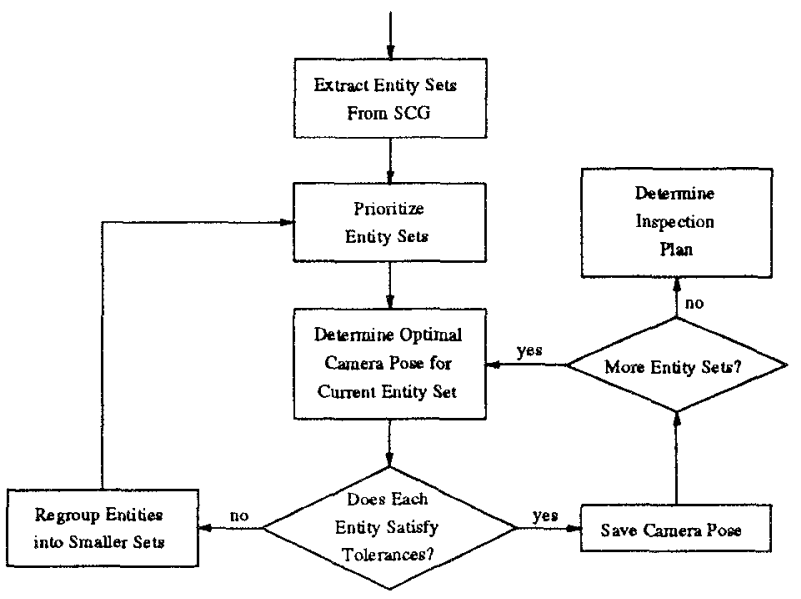

Figure 1: Flowchart embodying Visual Inspection Planning with Error Reduction (VIPER) system. The focus here is to determine optimal camera poses and to satisfy tolerance criteria.

the camera's capability in observing each entity. Griffin and Villalobos [3] have also modeled illumination errors.

However, unlike the previous works, the VIPER system determines an optimal camera pose based on the inherent inspection errors of displacement and quantization within given resolution, focus, field-of-view, and visibility constraints. Furthermore, unlike previous works, we formulate the probability that these errors are within the desired dimensional tolerances, which is necessary in evaluating the accuracy of the inspection.

In summary, the main contributions of this work are the following:

- Formulate the mean squared error of the combined displacement and quantization errors for line entities, taking into account all dependencies.

- Develop a method for obtaining camera poses where the minimum mean squared error (MMSE) is obtained within the sensor constraints of resolution, focus, fieldof-view, and visibility.

- Analytically derive the characteristic function for the total error. This function is used to determine if the probability of errors being in an acceptable range meets or exceeds a required threshold. The acceptable range is based on specified dimensional tolerances. 


\section{Modeling Errors}

Two important errors that cannot be avoided are displacement and quantization errors, both defined as the difference between the observed and actual lengths of the observed entities. The total error, $\varepsilon$ is the sum of the quantization and displacement errors.

To determine an optimal camera pose that minimizes the effects of these errors, the mean-squared error (MSE) is formulated and minimized. This formulation for a line entity is based on the derivation of Yang, et al [9].

\subsection{Displacement Error}

We need to relate the camera pose to the world coordinate system. Suppose the position of the camera in the world coordinate system is given by $\vec{t}=\left(t_{x}, t_{y}, t_{z}\right)$, and its orientation is given by the parameters $(\phi, \theta, \psi)$. Assuming that $\vec{d}=\left(d_{x}, d_{y}, d_{z}\right)$ provides the position of the origin of the world coordinates in the camera coordinate system, the relationship between $\vec{d}$ and $\vec{t}$ is $\vec{d}=-R \vec{t}$, where $R$ is a $3 \times 3$ rotation matrix between the camera and world coordinate system. Using homogeneous coordinates, a world coordinate $(x, y, z)$ is related to an image plane coordinate $(u, v)$ by $(1)$, where $P$ is a typical perspective matrix.

$$
\left[\begin{array}{c}
c u \\
c v \\
c
\end{array}\right]=P Q\left[\begin{array}{c}
x \\
y \\
z \\
1
\end{array}\right], \text { where } Q=\left[\begin{array}{cccc}
r_{11} & r_{12} & r_{13} & d_{x} \\
r_{21} & r_{22} & r_{23} & d_{y} \\
r_{31} & r_{32} & r_{33} & d_{z} \\
0 & 0 & 0 & 1
\end{array}\right]
$$

Unfortunately, we can never get the robot arm exactly where we want. There are errors in the positioning of each of the six degrees of freedom. Define the differential matrix of (2). The elements of the differential matrix, $d x, d y, d z, d \phi, d \theta$, and $d \psi$ are independent random variables, typically modeled as Gaussian distributions with zero mean. The transformation that accounts for the errors is $Q^{\prime}=Q+\Delta Q$, and the new relationship between the coordinate frames becomes ( 3 ).

$$
\begin{aligned}
\Delta= & {\left[\begin{array}{cccc}
0 & -d \phi & d \theta & d x \\
d \phi & 0 & -d \psi & d y \\
-d \theta & d \psi & 0 & d z \\
0 & 0 & 0 & 0
\end{array}\right] } \\
& {\left[\begin{array}{c}
c^{\prime} u^{\prime} \\
c^{\prime} v^{\prime} \\
c^{\prime}
\end{array}\right]=P Q^{\prime}\left[\begin{array}{l}
x \\
y \\
z \\
1
\end{array}\right] }
\end{aligned}
$$

By defining the camera coordinates as in (4), we can solve for $(u, v)$ and $\left(u^{\prime}, v^{\prime}\right)$, which are given in (5) and (6).

$$
\begin{aligned}
C_{1}= & r_{11} x+r_{12} y+r_{13} z+d_{x} \\
C_{2}= & r_{21} x+r_{22} y+r_{23} z+d_{y} \\
C_{3}= & r_{31} x+r_{32} y+r_{33} z+d_{z} \\
& u=\frac{f C_{1}}{f-C_{3}} \\
& v=\frac{f C_{2}}{f-C_{3}} \\
u^{\prime}= & \frac{f\left(C_{2}+C_{3} d \theta-C_{2} d \phi+d x\right)}{f-\left(C_{3}+C_{2} d \psi-C_{1} d \theta+d z\right)} \\
v^{\prime}= & \frac{f\left(C_{2}+C_{1} d \phi-C_{3} d \psi+d y\right)}{f-\left(C_{3}+C_{2} d \psi-C_{1} d \theta+d z\right)}
\end{aligned}
$$

The displacement error in the horizontal and vertical directions of a single point as defined by Yang, et al [9] are given in (7), where $\lambda_{h}$ for $h=1, \ldots, 21$ are listed in (8). We would like to find the means, variances, and covariances of $\varepsilon_{d_{u}}$ and $\varepsilon_{d_{y}}$. Unfortunately, these properties cannot be expressed in closed form. However, if we consider the representation of (9), the moments of the Gaussian numerators and denominators are determined exactly, and an approximation to the moments of $\varepsilon_{d_{v}}$ and $\varepsilon_{d_{v}}$ can be obtained.

$$
\begin{array}{rlrl}
\varepsilon_{d_{u}} & =u^{\prime}-u & \\
& =\frac{\left[\lambda_{1} \lambda_{2} \lambda_{3} \lambda_{4} \lambda_{5} \lambda_{6} \lambda_{7}\right][d \psi d \theta d \phi d x d y d z 1]^{T}}{\left[\lambda_{15} \lambda_{16} \lambda_{17} \lambda_{18} \lambda_{19} \lambda_{20} \lambda_{21}\right][d \psi d \theta d \phi d x d y d z 1]^{T}} \\
\varepsilon_{d_{v}} & =v^{\prime}-v & & \\
& =\frac{\left[\lambda_{8} \lambda_{9} \lambda_{10} \lambda_{13} \lambda_{12} \lambda_{13} \lambda_{14}\right][d \psi d \theta d \phi d x d y d z 1]^{T}}{\left[\lambda_{15} \lambda_{16} \lambda_{17} \lambda_{18} \lambda_{19} \lambda_{20} \lambda_{21}\right][d \psi d \theta d \phi d x d y d z 1]^{T}} \\
& & & \\
\lambda_{1} & =f C_{1} C_{2} & \lambda_{2} & =f\left[\left(f-C_{3}\right) C_{3}-C_{1}^{2}\right] \\
\lambda_{3} & =f C_{2}\left(C_{3}-f\right) & \lambda_{4} & =f\left(f-C_{3}\right) \\
\lambda_{5} & =0 & \lambda_{6} & =f C_{1} \\
\lambda_{7} & =0 & \lambda_{8} & =f\left[C_{2}^{2}-\left(f-C_{3}\right) C_{3}\right] \\
\lambda_{9} & =-f C_{1} C_{2} & \lambda_{10} & =f C_{1}\left(f-C_{3}\right) \\
\lambda_{11} & =0 & \lambda_{12} & =f\left(f-C_{3}\right) \\
\lambda_{13} & =f C_{2} & \lambda_{14} & =0 \\
\lambda_{15} & =-C_{2}\left(f-C_{3}\right) & \lambda_{16} & =C_{1}\left(f-C_{3}\right) \\
\lambda_{17} & =0 & \lambda_{18} & =0 \\
\lambda_{19} & =0 & \lambda_{20} & =f-C_{3} \\
\lambda_{21} & =\left(f-C_{3}\right)^{2} & &
\end{array}
$$

$$
\begin{aligned}
& \varepsilon_{d_{x}}=\frac{s}{x} \\
& \varepsilon_{d_{v}}=\frac{s}{x}
\end{aligned}
$$

Define $M_{6}$ and $C_{6}$ to be the mean vector and covariance matrix for the six degrees of freedom, and define $J_{6}$ be the Jacobian matrix relating these errors to each of the random variables $\zeta, \xi$, and $\chi$. The means of $\zeta, \xi$, and $\chi$ are 0,0 , and $\lambda_{21}$, respectively, and are used to construct the mean vector $M_{d i v}$. Their covariance matrix is $C_{d i v}=J_{6} C_{6} J_{6}^{T}$.

Applying a second order Taylor series expansion to $g(x, y)=x / y$ about the means $\left(\eta_{x}, \eta_{y}\right)$, where $\eta_{x}=0$ and $\eta_{y} \gg 0$, we can estimate the mean $\eta_{g}$ and variance $\sigma_{g}^{2}$ by $(10)$.

$$
\begin{aligned}
\eta_{g} & \approx-\frac{r_{x y} \sigma_{x} \sigma_{y}}{\eta_{y}^{2}} \\
\sigma_{g}^{2} & \approx \frac{\sigma_{x}^{2}}{\eta_{y}^{2}}
\end{aligned}
$$

Define the Jacobian, $J_{d i v}$, relating the inputs $\zeta, \xi$, and $\chi$ to the outputs $\varepsilon_{d_{u}}$ and $\varepsilon_{d_{v}}$ by taking all partial derivatives of the relations in (9) for each entity endpoint, and evaluating at the means in $M_{d i v}$. For $\varepsilon_{d_{v}}$ and $\varepsilon_{d_{v}}$, the mean vector, $M_{u v}$, is constructed from (10), and the covariance matrix is $C_{u v}=J_{d i v} C_{d i v} J_{d i v}^{T}$, which is equivalent to a second order Taylor series expansion.

The displacement errors in the horizontal and vertical directions of a line entity as defined by Yang, et al [9] are given by $(11)$, where $\left(u_{1}, v_{1}\right)$ and $\left(u_{2}, v_{2}\right)$ are entity endpoints. The moment matrices, $M_{x y}$ and $C_{x y}$, are given in (12). The Jacobian, $J_{u v}$, embodying (11) is exact, since the relationships are linear. 


$$
\begin{aligned}
\varepsilon_{d_{x}} & =\varepsilon_{d_{u_{1}}}-\varepsilon_{d_{v_{2}}} \\
\varepsilon_{d_{y}} & =\varepsilon_{d_{v_{1}}}-\varepsilon_{d_{v_{2}}} \\
M_{x y} & =J_{u v} M_{u v} \\
C_{x y} & =J_{u v} C_{u v} J_{u v}^{T}
\end{aligned}
$$

The angle between the line entity and horizontal axis of the image plane is defined by $\gamma$ in (13) and is depicted by Figure 2. The resultant displacement error, $\varepsilon_{d}$, is geometrically approximated by Yang, et al as (14). Using Jacobian $J_{x y}$ to describe (14), the mean and covariance matrices, $M_{d}$ and $C_{d}$, respectively, are shown in (15).

$$
\tan \gamma=\frac{v_{1}-v_{2}}{u_{1}-u_{2}}
$$

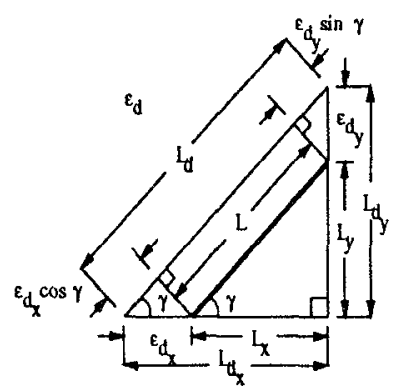

Figure 2: Graphical depiction of the approximation representing the displacement error, $\varepsilon_{d}$, for a line entity $L$. This approximation assumes that nominal and displaced entities are approximately parallel. (Figure not to scale. $\varepsilon_{d_{x}}$ and $\varepsilon_{d_{y}}$ are much smaller.)

$$
\begin{gathered}
\epsilon_{d} \approx \varepsilon_{d_{x}} \cos \gamma+\varepsilon_{d_{y}} \sin \gamma \\
M_{d}=J_{x y} M_{x y} \\
C_{d}=J_{x y} C_{x y} J_{x y}^{T}
\end{gathered}
$$

The total displacement error is the sum in (16), where $k$ is the number of line entities to observe. The mean and variance of $\varepsilon_{d}$ are obtained by summing all of the elements of $M_{d}$ and $C_{d}$, respectively. These moments and the displacement MSE are shown in (17).

$$
\begin{aligned}
\varepsilon_{d}=\sum_{j=1}^{k} \varepsilon_{d_{j}} \\
\eta_{\varepsilon_{d}}=\sum_{j=1}^{k} M_{d_{j}} \\
\sigma_{\epsilon_{d}}^{2}=\sum_{i=1}^{k} \sum_{j=1}^{k} C_{d_{i j}} \\
E\left[\varepsilon_{d}^{2}\right]=\sigma_{\epsilon_{d}}^{2}+\eta_{\varepsilon_{d}}^{2}
\end{aligned}
$$

\subsection{Quantization Error}

For the quantization error, the density functions for a line entity in two dimensions have been derived by Yang, et al [9]. For a single line entity in two dimensions, the actual and observed lengths are $L=\sqrt{L_{x}^{2}+L_{y}^{2}}$ and $L_{q}=\sqrt{L_{q_{x}}^{2}+L_{q_{y}}^{2}}$, respectively. Define $l_{x}$ and $l_{y}$ as the number of pixels completely covered by the line components, and denote the pixel dimensions as $r_{x}$ by $r_{y}$. The means, variances, and covariances are given in (18). If $\varepsilon_{q_{x}}=L_{q_{x}}-L_{x}$ and $\varepsilon_{q_{y}}=L_{q_{y}}-L_{y}$, then $E\left[\varepsilon_{q_{x}}\right]=E\left[\varepsilon_{q_{y}}\right]=0, E\left[\varepsilon_{q_{x}}^{2}\right]=\frac{1}{6} r_{x}^{2}$ and $E\left[\varepsilon_{q_{y}}^{2}\right]=\frac{1}{6} r_{y}^{2}$.

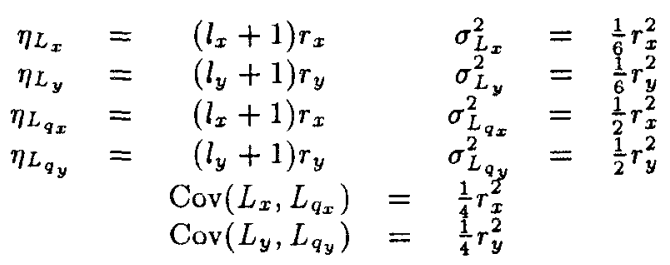

Geometrically approximating $\varepsilon_{q}$ in a manner analogous to Figure 2, as in (19), the mean and variance of the quantization error is (20). These results agree with [9].

$$
\begin{aligned}
\varepsilon_{q} & \approx \varepsilon_{q_{x}} \cos \gamma+\varepsilon_{q_{y}} \sin \gamma \\
E\left[\varepsilon_{q}\right] & \approx 0 \\
E\left[\varepsilon_{q}^{2}\right] & \approx \frac{1}{6}\left(r_{x}^{2} \cos ^{2} \gamma+r_{y}^{2} \sin ^{2} \gamma\right)
\end{aligned}
$$

For $k$ line entities, the total quantization error is given in (21), where each of the errors, $\varepsilon_{q_{j}}$, are independent. The moments of the total quantization error are determined by (22). Note that since $\eta_{\epsilon_{q}}=0$, then $E\left[\varepsilon_{q}^{2}\right]=\sigma_{\varepsilon_{q}}^{2}$.

$$
\begin{aligned}
\varepsilon_{q} & =\sum_{j=1}^{k} \varepsilon_{q_{j}} \\
\eta_{\varepsilon_{q}} & =\sum_{j=1}^{k} \eta_{q_{j}} \\
\sigma_{\varepsilon_{q}}^{2} & =\sum_{j=1}^{k} \sigma_{\varepsilon_{q_{j}}}^{2}
\end{aligned}
$$

\subsection{Combining Errors}

The combined displacement and quantization error for a line is determined by $\varepsilon=\varepsilon_{d}+\varepsilon_{q}$. Thus, the total MSE is (23), since $\varepsilon_{d}$ and $\varepsilon_{q}$ are independent.

$$
E\left[\varepsilon^{2}\right]=E\left[\varepsilon_{d}^{2}\right]+E\left[\varepsilon_{q}^{2}\right]
$$

\section{Sensor Constraints}

Define $\vec{r}_{O}$ and $\vec{\nu}$ to represent the position and viewing direction of the camera, respectively. We will need the conversions of (24) to relate with the transformation matrix in (1). Also, let $a$ be the lens aperture diameter, $d$ be the distance from the image plane and the back nodal point of the lens (effective focal length), $f$ be the lens focal length (intrinsic focal length). From Abrams, et al [1], $d$ can be determined from the focus constraint as (25), using the camera position $\left(\vec{r}_{O}\right)$ as well as the closest and furthest entity vertices along the viewing direction ( $\vec{r}_{c}$ and $\vec{r}_{f}$, respectively).

$$
\begin{aligned}
& \vec{r}_{O}=t_{x} \vec{\imath}+t_{y} \vec{\jmath}+t_{z} \vec{k} \\
& \vec{\nu}=r_{31} \vec{\imath}+r_{32} \vec{\jmath}+r_{33} \vec{k} \\
& d=\frac{2 D_{\max } f\left(D_{\max }-D_{f}\right)}{2 D_{\max }\left(D_{\max }-f-D_{f}\right)+D_{f}} \\
& \text { where } \\
& D_{\max }=\left(\vec{r}_{f}-\vec{r}_{O}\right) \cdot \vec{\nu} \\
& D_{f}=\left(\vec{r}_{f}-\vec{r}_{c}\right) \cdot \vec{\nu}
\end{aligned}
$$

The resolution and focus constraints are formulated by Tarabanis, et al [6], while the field-of-view constraint is 
reformulated. The planes defining the visibility constraints are extracted from an Entity-Based Aspect Graph (EAG), developed by Yang, et al [8]. All of the constraints are dependent on $\vec{r}_{O}$, and all but the visibility constraints are also dependent upon $\vec{\nu}$. Furthermore, each constraint can be defined for a set of entities.

The resolution constraint is formulated as (26), where $\vec{r}_{A}$ and $\vec{r}_{B}$ are the position vectors of the line entity vertices, $\vec{e}$ is the unit vector between them, $l$ is the length of the minimum entity to be resolved, and $w$ is the minimum required length of the image of the line. For each entity, there will be one resolution constraint.

$$
g_{1}: \frac{w}{d l}-\frac{\left\|\vec{\nu} \times\left[\vec{e} \times\left(\vec{r}_{O}-\vec{r}_{A}\right)\right]\right\|}{\left[\left(\vec{r}_{O}-\vec{r}_{A}\right) \cdot \vec{\nu}\right]\left[\left(\vec{r}_{O}-\vec{r}_{B}\right) \cdot \vec{\nu}\right]} \leq 0
$$

The focus constraints are defined by (27), where $\vec{r}_{c}$ and $\vec{r}_{f}$ are the position vectors of the closest and farthest entity vertices from $\vec{r}_{O}$ along the viewing direction, $D_{1}$ and $D_{2}$ are the far and near limits of the depth-of-field, respectively, given in (28), and $c$ is the radius of the blur circle. There will always be only two focus constraints for a set of entities.

$$
\begin{array}{cc}
g_{2 a}: & D_{2}-\left(\vec{r}_{c}-\vec{r}_{O}\right) \cdot \vec{\nu} \leq 0 \\
g_{2 b}: & \left(\vec{r}_{f}-\vec{r}_{O}\right) \cdot \vec{\nu}-D_{1} \leq 0 \\
& D_{1}=\frac{a f d}{a(d-f)-c f} \\
& D_{2}=\frac{a f d}{a(d-f)+c f}
\end{array}
$$

The field-of-view constraint is reformulated as (29), where $\vec{r}_{m}$ is the entity endpoint with the largest angle with $\vec{\nu}, \alpha$ is the field-of-view angle given in (30), and $I_{\min }$ is the minimum dimension of the image plane. There will always be one field-of-view constraint for any quantity of entities.

$$
\begin{gathered}
g_{3}:\left\|\vec{r}_{m}-\vec{r}_{O}\right\| \cos \left(\frac{\alpha}{2}\right)-\left(\vec{r}_{m}-\vec{r}_{O}\right) \cdot \vec{\nu} \leq 0 \\
\alpha=2 \tan ^{-1}\left(I_{\min } / 2 d\right)
\end{gathered}
$$

The visibility constraints are extracted from an EAG of the object. Each node in the EAG contains a subset of desired entities and is bounded by several planes of visibility. Hence, there would be several linear constraints (call them $g_{4 a}, g_{4 b}, \ldots$ ) corresponding to each set of entities, depending on the EAG node.

\section{Optimal Sensor Settings}

To determine an optimal camera pose, the minimum mean squared error (MMSE), $E\left[\varepsilon^{2}\right]$, is desired. Note that the mean and variance of the error are functions of the six degrees of freedom. Therefore, $E\left[\varepsilon^{2}\right]$ is a function of the six degrees of freedom as well. The sensor constraints are also defined by these parameters. We need to find a camera pose with an MMSE satisfying the sensor constraints.

Using methods of nonlinear programming with nonlinear constraints, $E\left[\varepsilon^{2}\right]$ can be minimized numerically so that resolution, field of view, focus, and visibility criteria are satisfied. This is formulated as (31), where $k$ represents the number of entities, $m$ is the number of half spaces in the visibility constraint, and $N(i)$ represents the ith letter of the alphabet ${ }^{1}$.

$$
\begin{aligned}
\operatorname{minimize} E\left[\varepsilon^{2}\right] & =f\left(t_{x}, t_{y}, t_{z}, \phi, \theta, \psi\right) \\
\text { subject to } g_{1 \kappa(i)} & \leq 0 \text { (resolution) for } i=1 \text { to } k \\
g_{2 a} & \leq 0 \text { (focus) } \\
g_{2 b} & \leq 0 \\
g_{3} & \leq 0 \text { (field-of-view) } \\
g_{4 \wedge(i)} & \leq 0 \text { (visibility) for } i=1 \text { to } \mathrm{m}
\end{aligned}
$$

\section{Dimensional Tolerances}

Due to imperfections in the inspection process, it is very possible that the length $L$ of an entity may deviate slightly from its nominal value. This deviation is modeled by a tolerance of $\pm \Delta L$. We would like to determine the probability that the error is within a specified deviation, and to compare this probability with an acceptable threshold. In other words, if the error of an entity is $\varepsilon$, then we need to determine if (32) holds, where $f_{\varepsilon}(\varepsilon)$ is the probability density function of the inspection error for a single line entity.

$$
\int_{-\Delta L}^{+\Delta L} f_{\varepsilon}(\varepsilon) d \varepsilon \geq \text { Threshold }
$$

Consider the characteristic function, $\Phi_{*}(\omega)$, related to $f_{\varepsilon}(\varepsilon)$ by (33). Reformulating (32) in terms of the characteristic function, (34) is obtained. To use these results, we must first determine the characteristic functions for the displacement and quantization errors.

$$
\begin{gathered}
f_{\epsilon}(\varepsilon)=\frac{1}{2 \pi} \int_{-\infty}^{+\infty} \Phi_{\epsilon}(\omega) e^{j \omega \epsilon} d \omega \\
\frac{1}{\pi} \int_{-\infty}^{+\infty} \frac{\Phi_{\epsilon}(\omega) \sin \omega \Delta L}{\omega} d \omega \geq \text { Threshold }
\end{gathered}
$$

Due to the complexity of the displacement error, it is advantageous to approximate its density with a simpler distribution. Recall that the horizontal and vertical errors for a point are defined as a ratio of two Gaussian RVs, $g(x, y)=x / y$, where $\eta_{x}=0$ and $\eta_{y}=\left(f-C_{3}\right)^{2}$, where $f$ is the focal length, and the camera coordinates $C_{1}, C_{2}$, and $C_{3}$ are defined in (4). As $C_{3}$ approaches $f$, the density approaches a Cauchy distribution. However, due to the focus constraints, the closest and furthest distances between the camera and the entity is $C_{3}=D_{2}$ and $C_{3}=D_{1}$, where $D_{1}$ and $D_{2}$ are given in (28) For any given $C_{3}$ within this range, we can define a range for the horizontal and vertical camera coordinates $C_{1}$ and $C_{2}$ based on the perspective projection of an image plane vertex onto the world coordinate frame, given in (35). These ranges prevent a Cauchy distribution from being realized.

$$
\begin{aligned}
& x=\frac{u(f-z)}{f} \\
& y=\frac{v(f-z)}{f}
\end{aligned}
$$

We have determined that within this range, the density of the ratio of two Gaussians may be effectively approximated with a Gaussian distribution. This is achieved by

\footnotetext{
${ }^{1}$ I.e. $\aleph(1)=a, \aleph(2)=b$, etc., so that $g_{4 N(1)}=g_{4 a}, g_{4 \times(2)}=$ $g_{4 b}$, etc.
} 
using the approximation in (10). When the mean and variance are applied to a Gaussian distribution model, actual density and its Gaussian approximate are indistinguishable, within these ranges. This has been verified by a number of simulations and plots. Thus, for our application, the ratio of Gaussian RVs may be approximated by another Gaussian RV.

Since all other random variables in the displacement error formulation are linearly related, the resultant displacement error will also have a Gaussian distribution. Expressing the displacement error of an entity with a Gaussian distribution, the characteristic function is given in (36), where $\eta_{\varepsilon_{d}}$ and $\sigma_{\epsilon_{d}}^{2}$ are components of $M_{d}$ and $C_{d}$ from (15).

$$
\Phi_{\varepsilon_{d}}(\omega)=e^{j \omega \eta_{\epsilon_{d}}-\frac{1}{2} \omega^{2} \sigma_{\epsilon_{d}}^{2}}
$$

Yang, et al [9] determined the distribution of the quantization error, based on uniformly distributed endpoint errors in both the horizontal and vertical directions. The characteristic functions for the horizontal and vertical quantization errors in a line are determined to be (37).

$$
\begin{aligned}
& \Phi_{\varepsilon_{q_{x}}}(\omega)=\frac{2}{\omega^{2} r_{x}^{2}}\left(1-\cos \omega r_{x}\right) \\
& \Phi_{\varepsilon_{q_{y}}}(\omega)=\frac{2}{\omega^{2} r_{y}^{2}}\left(1-\cos \omega r_{y}\right)
\end{aligned}
$$

Using (19), the final characteristic function of the quantization error is given in (38). The combined characteristic function is simply the product of (36) and (38), given in (39). Numerical integration of (34) then determines the probability that the inspection error of a line entity is within the tolerance of $\pm \Delta L$.

$$
\begin{gathered}
\Phi_{\varepsilon_{q}}(\omega)=\Phi_{\varepsilon_{q_{x}}}(\omega \cos \gamma) \Phi_{\varepsilon_{q_{y}}}(\omega \sin \gamma) \\
\Phi_{c}(\omega)=\Phi_{\varepsilon_{d}}(\omega) \Phi_{\varepsilon_{q}}(\omega)
\end{gathered}
$$

For each entity, the corresponding $\Delta L$ is determined based on the required accuracy and the length of the corresponding line segment projected onto the image plane using the assigned sensor setting. Given the coordinates of the entity's endpoints, the focal length, and the translational and orientational parameters of the sensor, the length of the projected line segment on the image plane, $L$, is computed. The tolerance, $\Delta L$, should be assigned based on $L$ and the minimum requirement of accuracy in the measurement. The tolerance is computed as described in (40).

$$
\Delta L=(1-\operatorname{accuracy}) L
$$

\section{Experimental Results}

Experiments were performed on the object in Figure 3, where each dimension is in decimeters (i.e. $4 \rightarrow 400 \mathrm{~mm}$ ). The optimizations in the experiments were performed by the GRG2 nonlinear programming package. The experiments use the following data as sensor and constraint parameters. The rotations were performed in the order $\phi$ about $z, \theta$ about $y$, and $\psi$ about $x$, with respect to the current coordinate axes.

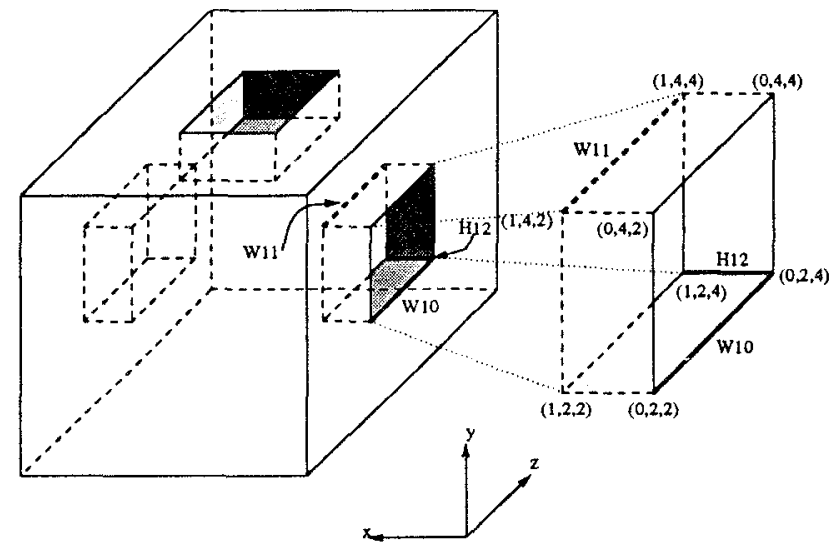

Figure 3: Object to inspect, including three line entities $W 11, H 12$, and $W 10$, and their world coordinates in decimeters. All experiments refer to these entities.

$$
\begin{aligned}
r_{x} & =0.01 \mathrm{~mm} \text { (horizontal pixel dim.) } \\
r_{y} & =0.013 \mathrm{~mm} \text { (vertical pixel dim.) } \\
f & =25 \mathrm{~mm} \\
a & =\frac{f}{16}=1.5625 \mathrm{~mm} \\
c & =r_{x} \text { (smallest pixel dim.) } \\
I_{\min } & =512 r_{x} \text { (smallest image plane dim.) } \\
I_{\max } & =480 r_{y} \text { (largest image plane dim.) } \\
w & =\sqrt{r_{x}^{2}+r_{y}^{2}} \text { (diagonal of pixel) } \\
l & =1 \mathrm{~mm} \\
\sigma_{x}^{2}=\sigma_{y}^{2}=\sigma_{z}^{2} & =0.03 \mathrm{~mm}^{2} \\
\sigma_{\phi}^{2}=\sigma_{\theta}^{2}=\sigma_{\psi}^{2} & =0.0003 \mathrm{rad}^{2}
\end{aligned}
$$

Experiment 6.1 An experiment was performed for the single entity $H 12$ of Figure 3 to test the effectiveness of the procedure described in this paper as applied to generating sensor settings. The results are given in Table 1 , where the camera pose ordering is $\left(t_{x}, t_{y}, t_{z}, \phi, \theta, \psi\right)$. The experiment completed after 108 iterations of the nonlinear program solver.

This experiment shows that the results agree with intuition. Notice that $H 12$ is readily visible from this position and viewing direction, since the camera is pointing toward the inside of the pocket. Thus, the success of the optimization is validated.

Experiment 6.2 To experiment with observing multiple entities from a single sensor setting, an experiment using both entities $H 12$ and $W 10$ of Figure 3 was performed, with the results given in Table 2 . The shown results were obtained after 349 iterations. A plot of the tolerance, $\Delta L$, against the probability for $H 12$ and $W 10$ are given in Figure 4, both using 100 integrations of (34).

This experiment shows that the final results make intuitive sense. As we might expect, the final MSE for observ- 
Table 1: Experimental results for inspecting entity $H 12$.

\begin{tabular}{|c|}
\hline \multicolumn{1}{|c|}{ Before Optimization: } \\
\hline Pose: $(-550,375,-900,0,0,0)$ \\
$\vec{v}:(0,0,1)$ \\
MSE: 0.00106117 \\
\hline \multicolumn{1}{|c|}{ After Optimization: } \\
\hline Pose: $(-535.33,397.53,-870.66,0.11197,0.41397,0.2050)$ \\
$\vec{\nu}:(0.414106,-0.158307,0.896357)$ \\
MSE: $1.734526 \times 10^{-5}$ \\
\hline
\end{tabular}

Table 2: Experimental results for inspecting both $\mathrm{H} 12$ and $W_{10}$, simultaneously.

\begin{tabular}{|c|}
\hline Before Optimization: \\
\hline Pose: $(-1500,750,-1700,-1.5708,0,-0.78540)$ \\
$\vec{\nu}:(0.707107,0,0.707107)$ \\
MSE: 0.000212734 \\
\hline After Optimization: \\
\hline Pose: $(-724.74,206.43,-481.53,-0.0060154,-2.3948,-3.1436)$ \\
$\vec{\nu}:(0.679287,-0.00607215,0.733847)$ \\
MSE: $3.641912 \times 10^{-5}$ \\
\hline
\end{tabular}

ing both $H 12$ and $W 10$ exceeds that of observing only $H 12$ from Table 1.

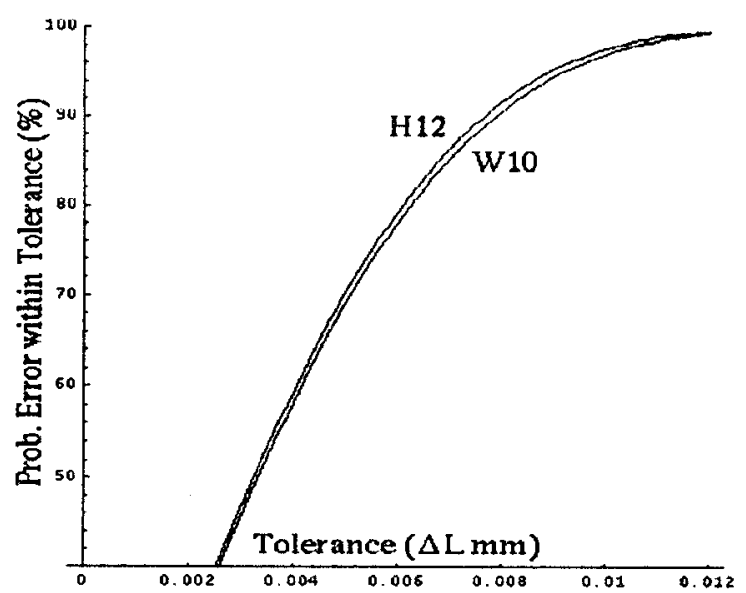

Figure 4: Plot of tolerance, $\Delta L$, versus probability that the total inspection error is within $\pm \Delta L$ for entities $H 12$ and $W 10$.

\section{Conclusion}

Dimensional inspection seeks to determine whether a manufactured object can be adequately inspected based on the dimensional tolerance specifications within a required threshold. It is important to determine a camera pose that reduces the impact of inherent errors, thus increasing the accuracy of such an inspection. In the VIPER system, we formulate the displacement error and quantization errors, taking into account all dependencies. By formulating and minimizing the mean squared error of the combined errors, we determine an optimal camera pose to best view the desired entities of an object. To obtain an effective camera pose, each observed entity must be resolvable, in focus, within the field of view, and unoccluded. Once these constraints are satisfied, we exploit the distribution of the sensing errors to determine the probability that the errors are within the specified tolerance. A comparison of this probability and the required threshold enables us to ascertain the acceptability and accuracy of the computed optimal camera pose. An expression for this probability has also been derived.

\section{Acknowledgments}

We thank Dr. Lasdon of the University of Texas and Dr. Waren of the Cleveland State University for providing a copy of the GRG2 software.

This research has been supported by the National Science Foundation under the grant IRI-9414523 to Dr. Marefat. The provided support is gratefully appreciated.

\section{References}

[1] Abrams, S., Allen, P. K., and Tarabanis, K. A., "Computing Camera Viewpoints in a Robot Work-Cell," Proceedings of the 1996 IEEE International Conference on Robotics and Automation, Vol. 3, pp. 1972-1979, Minneapolis, Minnesota, April 22-28, 1996.

[2] Cowan, C. K. and Kovesi, P. D., "Automatic Sensor Placement from Vision Task Requirements," IEEE Transactions on Pattern Analysis and Machine Intelligence, Vol. 10, No. 3, pp. 407-416, May 1988.

[3] Griffin, P. M. and Villalobos, J. R., "Process Capability of Automated Visual Inspection Systems," IEEE Transactions on Systems, Man, and Cybernetics, Vol. 22, No. 3 Pp. 441-448, May/June 1992.

[4] Niepold, R., Sakane, S., Sato, T., and Shirai, Y., "Vision Sensor Set-up Planning for a Hand-eye System using Environmental Model," Proceedings Society Instrum. Control Engineering, Japan, pp. 1037-1040, 1988.

[5] Sakane, S., Sato, T., "Automatic Planning of Light Source and Camera Placement for an Active Photometric Stereo System," Proceedings of IEEE International Conference on Robotics and Automation, pp. 1080-1087, Sacramento, California, April 1991.

[6] Tarabanis, K., Tsai, R. Y., and Allen, P. K., "The MVP Sensor Planning System for Robotic Vision Tasks," IEEE Transactions on Robotics and Automation, Vol. 11, No. 1, pp. 72-85, February 1995.

[7] Tarabanis, K., Tsai, R. Y., and Kaul, A., "Computing Occlusion-Free Viewpoints," IEEE Transactions on Pattern Analysis and Machine Intelligence, Vol. 18, No. 3, pp. 279-292, March 1996.

[8] Yang, C. C., Johnson, E. J., and Marefat, M. M., "EntityBased Aspect Graphs: Efficient Viewer Centered Representations," TR-ISL-96-9, Department of Electrical and Computer Engineering, University of Arizona, 1996.

[9] Yang, C. C., Marefat, M. M., and Ciarallo, F. W., "Analysis of Errors in Dimensional Inspection Based on Active Vision," Proceedings of SPIE, Intelligent Robots and Computer Vision XIII: $3 D$ Vision, Product Inspection, and Active Vision, pp. 96-104, Boston, Massachusetts, November $2-4,1994$.

[10] Yi, S., Haralick, R. M., and Shapiro, L. G., "Automatic Sensor and Light Source Positioning for Machine Vision," Proceedings 10th International Conference on Pattern Recognition, pp. 55-59, 1990. 